\title{
Pneumo-thorax/mediastinum/(retro)peritoneum/ scrotum - a full house of complications following JET ventilation
}

\author{
Pneumotorace/pneumomediastino/pneumoretroperitoneo/pneumoscroto - \\ complicanze a seguito di ventilazione JET
}

T.F. PÉZIER, G.-M. WIDMER, G.F. HUBER

ENT Clinic, University Hospital Zurich, Switzerland

\begin{abstract}
SUMMARY
We present a patient who presented to our clinic with airway obstruction secondary to oropharygeal cancer. He underwent emergent tracheostomy with JET ventilation, the latter resulting in a "full house" of barotraumatic complications including pneumothorax, pneumomediastinum, pneumoperitoneum, pneumoretroperitoneum and pneumo-scrotum. Free air, while sometimes dramatic as in our case, need not always be a cause for alarm and can often be managed expectantly. Our patient was treated with only a chest drain and otherwise made an uneventful recovery.
\end{abstract}

KEY WORDS: Jet ventilation $\bullet$ Chest drain $\bullet$ Pneumothorax $・$ Pneumomediastinum $\bullet$ Pneumoperitoneum $\bullet$ Pneumoretroperitoneum • Pneumo-scrotum $\bullet$ Free air $\bullet$ Subcutaneous emphysema

\section{RIASSUNTO}

Presentiamo il caso di un paziente che è giunto alla nostra clinica per un'ostruzione respiratoria secondaria a un carcinoma orofaringeo. Il paziente è stato sottoposto a una tracheotomia in emergenza e a una ventilazione JET, che ha dato come conseguenza un insieme di complicanze barotraumatiche, fra le quali un pneumotorace, penumomediastino, pneumoperitoneo, pneumoretroperitoneo, e pneumoscroto. Tale tipologia di ventilazione, benché talvolta dalle conseguenze drammatiche, come nel nostro caso, non è per questo necessariamente da temere e può essere ben gestita. Il nostro paziente è stato trattato con un drenaggio toracico ed è andato incontro a un recupero completo.

PAROLE CHIAVE: Ventilazione Jet $\bullet$ Drenaggio toracico $\bullet$ Pneumotorace $\bullet$ Penumomediastino $\bullet$ Pneumoperitoneo $\bullet$ Pneumoretroperitoneo $\bullet$ Pneumoscroto $・$ Ventilazione $\bullet$ Enfisema sottocutaneo

\section{Introduction}

Free air is often the sign of a ruptured viscera and must be taken seriously. For example, in ENT practice, oesophageal rupture following rigid oesophagoscopy must be treated appropriately. Free air can, however, present in other settings where it does not always mandate intervention. Iatrogenic free air can often be managed conservatively or with only minimal intervention providing the patient is appropriately monitored.

\section{Case report}

The 55-year-old man had originally been treated for a right sided cT3N2b tonsillar cancer infiltrating the tongue base and vallecula with chemoradiotherapy followed by a staged neck dissection in 2002. After 5 year follow-up he had been discharged, only to present some 10 years after the initial therapy with a cT3N0M1 biopsy-proven recurrence. Despite the thoracic surgeons being confident of achieving a curative resection of the distant metastasis, from an ENT point of view, the primary was unresectable and he had already received his maximal radiotherapy dose. He was therefore treated palliatively with cetuximab, cisplatin and 5-fluorouracil.

At his regular oncology appointment he was noted to have increasing airway compromise and sent for ENT consultation. On examination the patient showed stable cardiopulmonary conditions but with inspiratory stridor. Oral examination was almost impossible due to extreme trismus. Transnasal fibreendoscopy showed the epiglottis to be grossly swollen and contacting the posterior pharynx. No view beyond could be obtained.

The situation was discussed with the patient and the decision made for emergent tracheostomy. 
Before planned fibreoptic transnasal intubation, a Ravussin needle was inserted to improve oxygenation. The anaesthetic team however quickly spotted subcutaneous emphysema and the patient's breathing became worse. The decision was taken to perform an awake cricothyroidotomy under local anaesthesia. Once the cricothyroidotomy was completed, the patient was fully anaesthetised and the cricothyroidotomy converted to a surgical tracheotomy.

Post-operatively, the patient continued to have breathing difficulties and chest auscultation revealed reduced breath sounds on the right together with a hyper-expanded and hyper-resonant right chest. Chest X-ray (Fig. 1) confirmed clinical diagnosis of a pneumothorax and $20 \mathrm{ch}$ chest drain was placed in standard fashion. The chest Xray also showed bilateral sub-diaphragmatic free air and pneumo-mediastinum. Repeat chest X-ray showed appropriate placement of the chest drain and the lung to have re-expanded (not shown).

Clinically, the patient's cardiovascular status was stable with no signs of engorged neck veins. Furthermore, the abdomen was soft with normal bowel sounds. Scrotal examination showed an enlarged and tender scrotum and the clinical diagnosis of pneumo-scrotum was made. The chest drain was swinging normally without any bubbles. Blood tests revealed mild leukocytosis and C-reactive protein of 85 . To better evaluate the extent of mediastinal/ abdominal/ scrotal free air, a triple contrast chest/abdominal CT. This showed copious bilateral subcutaneous emphysema, small pneumothoraces bilaterally, mediastinal, intra-abdominal, retroperitoneal and scrotal free air (Figs. 2, 3).

Regular general surgical and thorax surgical consults

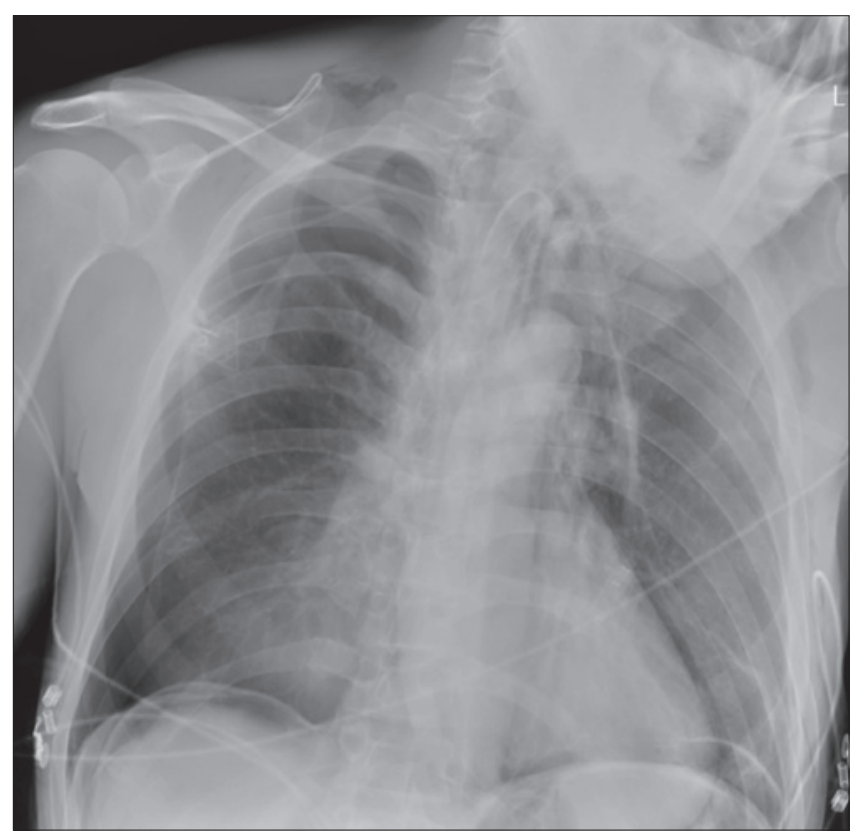

Fig. 1. Chest $\mathrm{X}$-ray showing large right sided pneumothorax, pneumo-mediastinum and bilateral sub-diaphragmatic free air.

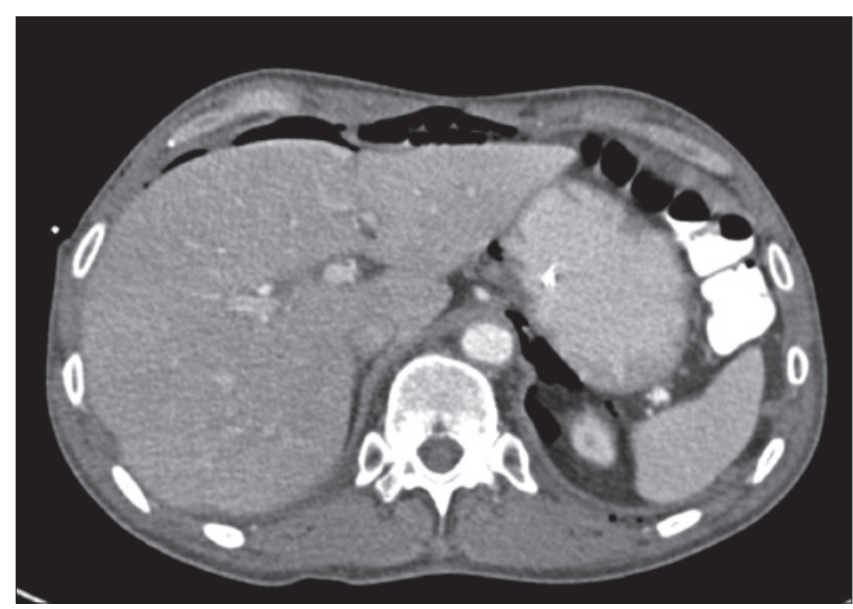

Fig. 2. Axial slice triple contrast chest/abdominal CT at the level of the right adrenal gland showing intra-abdominal and retroperitoneal free air.

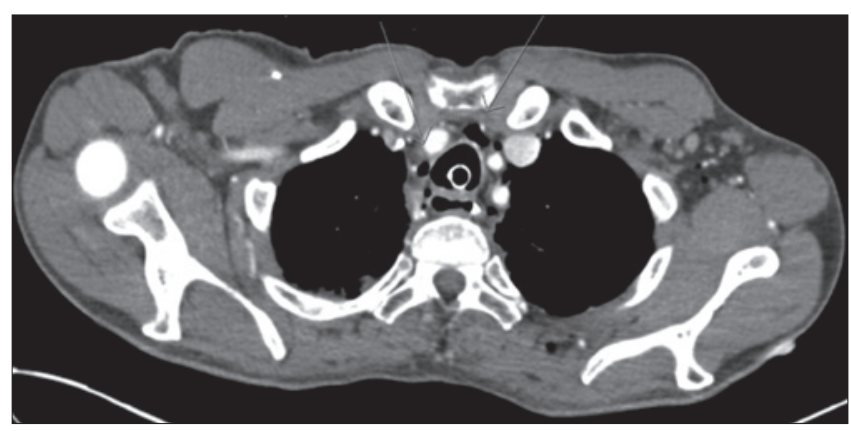

Fig. 3. Axial slice triple contrast chest/abdominal CT showing mediastinal free air (arrows) and subcutaneous emphysema on the left.

were made in the following days and the patient remained clinically stable. The chest drain was removed on post-operative day 5. Clinically the subcutaneous emphysema and scrotal air resolved over the following days such that the patient could be discharged back to oncological care.

\section{Discussion}

\section{Barotrauma}

Pulmonary barotrauma is a recognised complication of mechanical ventilation, especially in neonates. Other patients thought to be particularly at risk include asthmatics and those with chronic interstitial lung disease or acute respiratory distress syndrome (ARDS).

Positive pressure ventilation results in an elevated transalveolar pressure and can lead to alveolar rupture and air escape ${ }^{1}$. Previously, it was estimated that roughly $10 \%$ of patients undergoing mechanical ventilation suffered barotrauma, but the increasing use of low tidal volume ventilation is thought to have reduced this. 
The escaping then air dissects along tissue planes. Two theories explain how the air can exit the thoracic compartment: (a) direct passage through pleural and diaphragmatic defects and/or (b) via perivascular connective tissue to the retroperitoneum and finally to the peritoneum ${ }^{2}$. Serious complications including tension pneumothorax, tension pnemo-mediastinum and tension pneumo-peritoneum have been reported.

Several ventilation strategies have been suggested to reduce the risk of barotrauma. Reducing the peak airway pressure seems to have little effect, although reducing plateau airway pressures (a more accurate measure of alveolar pressure) to $<35 \mathrm{~cm} \mathrm{H}_{2} \mathrm{O}$ is effective.

\section{Needle cricothyroidotomy ventilation}

Needle cricothyroidotomy (NC) was first described in the $1950 \mathrm{~s}^{3}$ and can be used to temporalise a critical airway until more definitive measures are taken. This has the benefit of achieving oxygenation without requiring sedation or muscle relaxant. Indeed, a similar technique $\left(\mathrm{SCOOP}^{\circledR}\right)$ is often used by chronic obstructive pulmonary disease patients for long-term oxygen therapy. Oxygen can be delivered at continuous positive pressure or via high frequency, low tidal volume ventilation (JET) ${ }^{4}$.

Needle cricothyroidotomy has proven itself invaluable in "can't intubate, can't ventilate scenarios" for example following trauma or gross swelling of the upper airway from infection/allergy/chemical or thermal burns. However, the technique is not without complications, which include possible barotrauma, hypercapnia and ongoing aspiration risk. Contraindications to $\mathrm{NC}$ include situations where the airway can safely be maintained with non-invasive measures. An assessment must also be made of an effective "chimney" for the air to escape through the mouth and nose. Indeed, in normal patients, up to a third of the insufflated air is thought to escape superiorly without ever passing through the lungs. An inadequate chimney, for example in the setting of upper airway obstruction, can result in massive distention of the lungs and barotrauma ${ }^{5}$. This complication can be mitigated by using prolonged expiratory times (I:E ratio 1:10 at a respiratory rate of 5-6/ minute $)$, a lower insufflation pressure $(<350 \mathrm{kPa}$ in adults and $<200 \mathrm{kPa}$ in children) and a larger internal diameter catheters.

The risk of hypercapnia due to inadequate ventilation despite adequate oxygenation is thought to result within 45 min ${ }^{6}$. In certain circumstance however, hypercapnia may be tolerated though particular attention should be paid to possible rising intracranial pressure.

It should finally be emphasised that NC does not protect the airway. Aspiration risk can be considerable, especially in the setting of trauma and bleeding. The position of the needle in the trachea should mean, however, a very low risk of insufflating air into the stomach.

\section{Management of complications}

Herien, we focus on the complications of barotrauma outside of the lungs. Our patient had bilateral pneumothoraces, pneumo-mediastinum, pneumo-peritoneum/ retroperitonuem/scrotum.

\section{Pneumothorax}

Close observation of small pneumothoraces is acceptable, but larger pneumothoraces, or those progressing to airway compromise or tension will require needle decompression and definitive chest drain insertion. Smaller drains are generally easier to insert and are more comfortable for the patient and are as efficacious as larger drains ${ }^{7}$. Chest tubes should be connected to an underwater seal, normally without suction (to avoid re-expansion pulmonary oedema ${ }^{8}$ ). Failure of re-expansion or persistent leak can be managed with video-assisted thorascopic surgery with/without pleurodesis or open thoracostomy. Once no longer bubbling, the drain may be clamped for a period of observation (often including check chest X-ray) before being removed.

\section{Pneumo-mediastinum}

Pneumo-mediastinum can also be managed expectantly, though rarely patients will develop tension pneumo-mediastinum which can be similarly be managed acutely with needle decompression followed by mediastinotomy and tube placement. Loss of cardiac output due to reduced venous return with bulging neck veins are worrying signs which should prompt immediate cardio-thoracic consultation.

\section{Pneumoperitoneum}

Pneumoperitoneum is a recognised complication of a perforated abdominal viscera, but can occur in settings without obvious perforation. Clearly, post-abdominal procedure free air is a normal finding, but free air can sometimes also be seen post-colonoscopy or secondary to transvaginal procedures or intestinal Pneumatosis cystoides infection. The management of these latter causes of pneumo-peritoneum is patient specific and depends on clinical findings, vital parameters and blood results. Often a conservative management can be tried before explorative laparoscopy/laparotomy.

Following barotrauma, pneumo-peritoneum is usually self-limiting with $97 \%$ of cases resolving within 5 days ${ }^{9}$. Occasionally patients can suffer abdominal compartment syndrome and require intervention.

\section{Conclusions}

Needle cricothyroidotomy is an invaluable weapon in the anaesthetist's armamentarium, but is not risk-free and can only temporalise an airway. Pulmonary baro- 
trauma is a real possibility and appears to increase intensive care length of stay and mortality ${ }^{10}$. Anaesthesiologists, intensive care personnel and other staff need to be aware of what method of airway management was used intra-operatively in order to be appropriately vigilant post-operatively and recognise findings in the appropriate context. Free air, while sometimes dramatic as in our case, need not always be a cause for alarm and can often be managed expectantly.

\section{References}

1 Parker JC, Hernandez LA, Peevy KJ. Mechanisms of ventilator-induced lung injury. Crit Care Med 1993;21:131-43.

2 Macklin C. Transport of air along sheaths of pulmonic blood vessels from alveoli to mediastinum: Clinical Implications. Arch Intern Med 1939;64:913-26.

3 Jacoby JJ. Simple method of artificial respiration. Am J Physio 1951;167:798.

4 Ihra G, Gockner G, Kashanipour A, et al. High-frequency jet ventilation in European and North American institutions: developments and clinical practice. Eur J Anaesthesiol 2000;17:418-30.

5 Neff CC, Pfister RC, Van Sonnenberg E. Percutaneous transtracheal ventilation: experimental and practical aspects. $\mathrm{J}$ Trauma 1983;23:84-90.

6 Mace SE, Khan N. Needle cricothyrotomy. Emerg Med Clin N Am 2008;26:1085-101, xi.

7 Tsai WK, Chen W, Lee JCet al. Pigtail catheters vs largebore chest tubes for management of secondary spontaneous pneumothoraces in adults. Am J Emerg Med 2006;24:795-800.

8 MacDuff A, Arnold A, Harvey J, Group BTSPDG. Management of spontaneous pneumothorax: British Thoracic Society Pleural Disease Guideline 2010. Thorax 2010;65(Suppl 2):ii18-31.

9 Nielsen KT, Lund L, Larsen LP, et al. Duration of postoperative pneumoperitoneum. Eur J Surg 1997;163:501-3.

10 Anzueto A, Frutos-Vivar F, Esteban A, et al. Incidence, risk factors and outcome of barotrauma in mechanically ventilated patients. J. Intensive Care Med 2004;30:612-9.

Received: March 16, 2015 - Accepted: October 19, 2015

Address for correspondence: Thomas F. Pézier, ENT Clinic, University Hospital Zurich, Frauenklinikstrasse 24, 8032 Zurich, Switzerland. E-mail: tfrpezier@gmail.com 